\section{Cahiers de Narratologie}

Analyse et théorie narratives

12 | 2005

Récit et éthique

\title{
La théorie sémiotique littéraire de Michael Riffaterre : matrice, intertexte et interprétant
}

John Hopkins

\section{(2) OpenEdition}

\section{Journals}

Édition électronique

URL : https://journals.openedition.org/narratologie/37

DOI : 10.4000/narratologie.37

ISSN : 1765-307X

Éditeur

LIRCES

\section{Référence électronique}

John Hopkins, «La théorie sémiotique littéraire de Michael Riffaterre : matrice, intertexte et interprétant », Cahiers de Narratologie [En ligne], 12 | 2005, mis en ligne le 20 avril 2005, consulté le 04 mars 2022. URL : http://journals.openedition.org/narratologie/37 ; DOI : https://doi.org/10.4000/ narratologie.37

Ce document a été généré automatiquement le 4 mars 2022.

Article L.111-1 du Code de la propriété intellectuelle. 


\title{
La théorie sémiotique littéraire de Michael Riffaterre : matrice, intertexte et interprétant
}

\author{
John Hopkins
}

1 Cette communication présentée lors d'un séminaire du CNA, présente une vision de la théorie littéraire de Michael Riffaterre et propose d'en compléter la portée par un apport théorique nouveau. La dernière partie examine les rapports de la théorie riffaterrienne avec le roman.

2 Sémiotique, poésie, matrice, structure bimatricielle, Riffaterre, Baudelaire.

3 Puisque je parle aux confins du Centre de Narratologie Appliquée, où l'on s'intéresse principalement aux questions de la structure et de l'interprétation du récit, je dois m'excuser de vous tenir des propos sur la poésie, où le message s'organise plutôt de façon paradigmatique, ou non-linéaire. Il s'agira de la présentation et de la critique de la théorie herméneutique de la poésie élaborée par Michael Riffaterre qui -parmi tous ceux qui s'occupent de la théorie littéraire- cherche constamment à appliquer sa théorie à des textes poétiques. En effet, Riffaterre est connu comme le lecteur par excellence de la poésie française depuis Baudelaire.

4 Par où aborder le trajet du développement de cette théorie? Commençons par le fameux débat sur Les Chats, de Baudelaire, débat déclenché par l'analyse textuelle faite par l'équipe Jakobson / Lévi-Strauss, publiée en 1962 (voir Selected Writings III, 447-464). Très brièvement, la plupart de l'article est consacrée à une étude minutieuse poursuivie sans doute par Jakobson- d'abondants exemples de correspondances phonologiques ou syntaxiques existants dans le texte. Par la suite, il ne parvient pas à montrer en quoi ces exemples d'assonance, etc., contribuent à la signification globale du texte. Le soin de proposer une hypothèse sur cette dernière question revient à LéviStrauss -hypothèse faite vers la fin de l'article et qu'il néglige de relier aux détails phonologiques indiqués par Jakobson.

5 Dans sa réponse à l'article des deux collaborateurs célèbres, Riffaterre ne manque pas de souligner ce fait (dans son article de 1966, dans Yale French Studies; traduction 
française aux Essais de stylistique structurale, 1971). A propos des systèmes de correspondances établis par Jakobson, Riffaterre commente: "Les équivalences établies sur la base de ressemblances purement syntaxiques peuvent sembler particulièrement contestables" (Essais, p.316). Il en conclut qu' “aucune analyse grammaticale d'un poème ne peut nous donner plus que la grammaire du poème" (ibid., p.325).

La matrice

6 Dans une section de l'article à part, intitulée "Le poème comme un tout", Riffaterre annonce, déjà en 1966, l'essentiel d'une méthode qu'il développera par la suite, pour aboutir à son ouvrage le plus influent (sinon le meilleur connu du monde francophone), Semiotics of Poetry (1978, traduction française 1983). Avec la publication de ce livre, l'œuvre critique de Riffaterre - tout en retenant une base structuraliste- évolue vers une orientation sémiotique. Déjà, en 1966, Riffaterre oblige le lecteur à faire une relecture du texte entier - selon laquelle "la connaissance de tous les faits et de la fin agit rétroactivement pour modifier notre perception originelle du texte" (Essais, p.353). Il s'agit, plus ou moins, de la 'rétrolecture' de Riffaterre 1978, selon laquelle le lecteur s'aperçoit que tout un ensemble d'images d'un poème constituent en effet des variantes d'une seule proposition sous-jacente, qui les génère toutes. Cette "seule structure sémantique" mentionnée déjà en 1966 n'est rien d'autre que la matrice de 1978. Cette matrice - au sens d'une proposition sous-jacente génératrice de plusieurs images apparaissant sur la surface textuelle- n'est jamais actualisée en soi sur cette surface. Il reste au lecteur à la reformuler d'après une comparaison approfondie des images de la surface, visant à reconstituer leur structure propositionnelle commune.

7 Le fait que la matrice reste au sein même du texte - sans jamais être visible à sa surface - explique pourquoi tant de lecteurs de textes modernes, croyant pouvoir déchiffrer un poème selon les conventions du langage sociolectique de tous les jours, échouent devant l'opacité (en termes riffaterriens 'l'agrammaticalité') de cette surface. Car il s'agit de la poésie depuis Baudelaire, une poésie qui cherche à exprimer indirectement à travers un système d'images symboliques - ce que le poète ne pouvait pas exprimer directement. Sans l'aide de la structure sous-jacente qui génère ces symboles, beaucoup de lecteurs -y-compris des critiques renommés- sont tombés dans ce que Riffaterre appelle la 'referential fallacy' (l'illusion référentielle): ils se sont permis de croire que la structure superficielle des images d'un poème réfère directement à des phénomènes extra-textuels. Dans le cas des Chats, par exemple, Riffaterre observe que le titre même mènera certains à conclure qu'il s'agit d'un 'poème animalier'. Puisque avec Baudelaire nous sommes à l'aube du modernisme, selon Riffaterre, il reste la possibilité que, sur un niveau superficiel, on peut lire Les Chats "comme un blason...une laus cattorum", aussi bien que comme un poème symbolique. Ceci dit, il part à la recherche de la signification symbolique des Chats - voire de l'univers symbolique particulier à ce texte.

8 Riffaterre commence par souligner que "Dans tous les cas où les chats sont l'équivalent de quelque chose d'autre [...], ils ne ressemblent pas à l'apparence de ces équivalents mais à ce que ceux-ci représentent" (Essais, p.353). Pour Riffaterre, les images contribuant à établir leur matrice (qui sont donc générées par cette matrice) sont celles où les chats deviennent symboles de la "vie contemplative". Notons qu'il s'agit d'une expression qui ne se trouve nulle part sur la surface textuelle. Surtout les vers 9 à $11-$ où les chats empruntent les caractéristiques des "grands sphinx" voués à la méditation - contribuent à cette impression. Riffaterre est apparemment persuadé que cette matrice génère le texte en entier, à cause de la présence du mot sédentaires à la 
première strophe, que l'on pourrait associer à la posture des sphinx. Une telle conception n'expliquerait pas la notion d'activité impliquée par les expressions "amoureux fervents", "volupté", "coursiers" et "reins féconds" - une activité néfaste, si l'on ajoute les idées de "L'Erèbe" et les "ténèbres" qui s'y associent. Le vers 5 juxtapose "science" et "volupté" d'une façon qui suggère qu'elles s'équivalent au niveau sémiotique. Cette équation pourrait être générée par une autre matrice -que j'appellerai la première- qui engendrerait les deux premières stances, représentant les chats sous leur forme animale, comme des êtres dévoués au côté physique de l'existence plutôt que sous une forme figurative comme dans la deuxième matrice, qui génère les vers 9-14. Riffaterre lui-même semble reconnaître l'existence de deux pôles conceptuels dans le texte, puisqu'il parle d'un "shift", ou d'une "transformation" du rôle 'naturel' du chat à son rôle 'surnaturel': "Beneath this repetitive continuity [de la structure commune des images générées par le système unimatriciel qu'il propose] lies an antithesis that opposes the natural cat, symbol of contemplation, to the supernatural cat, symbol of the contemplated, of the occult truth" (Riffaterre 1966:234).

9 Il reste l'anomalie apparente des mots "doux" et "sédentaires" et peut-être "frileux", qui du moins semblent s'associer à la matrice 'contemplative'. Pourtant, ceux-ci sont tous des attributs des "savants": la science et la sensualité prennent toutes les deux la réalité physique comme objet de leurs 'recherches', par contraste avec l'objet métaphysique -idée commune aux mots "magiques" et "mystiques"-, de la vie contemplative de la deuxième matrice.

10 Dans un important article de 1969 (intitulé "Three models for the description of poetry") W.O. Hendricks corrobore l'analyse proposée ci-dessus du poème en deux parties générées chacune par deux concepts matriciels distincts. De plus, il observe qu'une interprétation unimatricielle, bien qu'il n'emploie pas ce terme, ne pourrait pas expliquer la progression syntagmatique des idées à travers le texte (Hendricks 1969:19). Quelles que soient les insuffisances de l'analyse de nos deux critiques, dont l'interprétation globale n'est pas reliée de façon convaincante au réseau de parallélismes formels distingués dans le texte, Jakobson et Lévi-Strauss néanmoins font une observation importante lorsqu'ils parlent d'une "progression dynamique du début à la fin [du texte]" (Selected Writings III:461). Les chats, proposent-ils, jouent un rôle médiateur entre "la constellation initiale du poème, formée par les amoureux et les savants" et une deuxième "constellation" - serait-ce un système matriciel?- où le poète parvient à s'unir à l'univers (interprétation attribuée par Georges Mounin à LéviStrauss).

11 Quoi que l'on pense de la caractérisation de ces deux 'constellations' dont la seconde s'écarte notablement de l'idée de la vie contemplative, Lévi-Strauss semble indiquer l'existence d'un phénomène équivalent à nos deux systèmes matriciels et d'un lien syntagmatique entre les deux. Étant donné que Riffaterre lui-même parait également être conscient de l'existence de cette structure, et du "shift" entre ses deux composants -impliquant une progression syntagmatique- il est surprenant qu'il soutienne dès le début de son analyse que le poème est généré par un seul concept matriciel. (Ajoutons entre parenthèses que la notion riffaterrienne de la matrice comme génératrice d'un ensemble d'images rappelle l'analyse proposée par Lévi-Strauss du mythe d'Oedipe, dans L'anthropologie structurale. Là, l'ethnologue distingue quatre éléments constitutifs $\mathrm{du}$ récit, chacun générant plusieurs variantes. Par exemple le concept-noyau du fratricide se développe dans la phrase quasi-matricielle de la 'négation de la parenté', 
qui se trouve à la base des variantes suivantes: 'Les Spartiates se tuent les uns les autres', 'Oedipe tue son père, Laios', etc..)

12 Quant au lien syntagmatique entre nos deux phrases matricielles, la première présente un contraste net avec la deuxième: tandis que l'image du sphinx et de sa vie contemplative isole de la réalité physique, le chat métaphysique apparaissant dans la seconde matrice (PM2), le chat de la première (PM1) est non seulement non pas métaphysique, il est super-physique. Notons que même la 'volupté' du chat de PM1 perd sa physicalité à PM2: ses reins ne sont plus chargés d'une énergie sexuelle ordinaire, mais d'étincelles magiques! Il semble donc vraisemblable de dire que la proposition faite par PM2 ("le chat symbolise la vie contemplative") -laquelle est plus innovantel'emporte sur celle de PM1, laquelle est plutôt proche du sociolecte. Il faut souligner d'ailleurs que ce mouvement syntagmatique -puisqu'il concerne la relation entre les deux structures sous-jacentes constituant la structure propositionnelle du poème- $a$ lieu au niveau sémiotique du texte: il n'entretient pas de relation nécessaire avec la progression syntaxique d'une lecture mimétique au niveau superficiel.

13 On peut dire que cette relation intermatricielle opère au niveau sémiotique parce que la matrice constitue - selon Riffaterre- l'essentiel du texte en tant que signe textuel. Puisque j'ai proposé qu'il existe deux matrices (et il semble qu'une structure semblable se trouve à la base de tout texte moderne), il s'agit d'un signe textuel complexe, constitué de deux paradigmes d'images reliées par une relation syntagmatique. Il fonctionne donc aux deux axes du langage signalés par Saussure. Remarquons que la matrice est une structure dans le sens du structuralisme classique: elle constitue une proposition-mère qui surdétermine la structure interne de chaque image du texte. Donc, à la dernière strophe de notre texte, les étincelles magiques des reins des chats comme les prunelles mystiques de leurs yeux- sont 'magiques' et 'mystiques' en fonction de la 2ème matrice selon laquelle les chats symbolisent la vie contemplative.

14 Cette conception du binarisme de la structure matricielle d'un texte poétique est soutenue par des remarques du critique Jonathan Culler à propos d'une analyse riffaterrienne du poème Fêtes de la faim, de Rimbaud. Face à une matrice de structure binaire proposée initialement par Riffaterre lui-même: "manger le comestible, manger l'immangeable"-proposition reniée par la suite-Culler suggère que "in many cases readings unify a poem with the aid of some elementary binary opposition" (Culler 1981:93). Bien que Culler ne donne pas d'exemples, un binarisme matriciel -ou bimatricialité- se trouve dans chaque texte analysé en entier par Riffaterre, dans une centaine de publications. Pourtant, il recourt à divers subterfuges pour éviter d'admettre l'existence d'une deuxième matrice, n'utilisant plusieurs fois qu'une partie du texte analysé.

Hypogramme et matrice

15 Considérons brièvement un autre concept essentiel de Semiotics of Poetry l'hypogramme- parce qu'il est assez souvent confondu avec la matrice. Ce terme est emprunté aux recherches de Saussure sur les anagrammes en poésie latine. Chez Riffaterre il n'est rien de plus qu'une phrase (un lieu-commun, un cliché) préexistante dans le sociolecte, dont le lecteur se rappelle en lisant une image poétique contenant un vocabulaire semblable. L'hypogramme donne donc au lecteur une impression de déjà-vu. Si une image rappelle un hypogramme quelconque ou non, elle ne peut faire fonction de signe poétique dans un texte que si elle est modelée sur la structure lexicosyntaxique d'une matrice génératrice de tout un ensemble d'images. Il se peut que 
plusieurs images d'un ensemble partagent un matériau lexical commun, qui se réfère à un même système descriptif hypogrammatique.

16 Puisque l'hypogramme n'a aucune relation nécessaire avec la matrice sur le plan syntaxique, consistant plutôt en un ensemble de vocables rappelés par ceux des images textuelles, il a plus en commun avec le paragramme saussurien. Celui-ci consiste en des fragments d'un mot-clé qui se trouvent incorporé à d'autres mots éparpillés à travers le texte. (L'hypogramme de Saussure constitue simplement la forme originale de ce mot c'est-à-dire la forme reconstituée par le lecteur à partir de sa forme paragrammatique.) Pour Riffaterre le coup de génie de Saussure réside avant tout dans sa perception de cette nécessité de reconstituer une structure qui n'apparaît pas sous la même forme dans le texte. A le citer: "Saussure's genius was to understand that the text's true centre is outside the text" (Rif.1974b:72-de l'article "Paragram and Significance"). Il est clair que Riffaterre songe ici à un concept voisin de sa matrice.

\section{L'intertexte}

17 Il faut souligner que toute tentative de formuler la matrice d'un texte reste sur une hypothèse faite par le lecteur. Mais, dans la théorie riffaterrienne, la structure de la matrice a pour autorité une structure intertextuelle analogue, que le lecteur est obligé de chercher dans d'autres textes (ou oeuvres d'art comme des tableaux, des films) de sa connaissance. Selon les modifications que j'ai apportées à la théorie, il s'agira d'une structure intertextuelle bimatricielle dont la relation intermatricielle sera analogue à celle du texte. Soulignons que l'intertexte opère sur le plan de la structure du texte entier: il ne s'agit point de relations intertextuelles entre une partie ou l'autre du texte primaire et d'autres textes. Plusieurs critiques se sont trompés sur ce point, dont le Genette de Palimpsestes (1982), qui nous dit que "les rapports (intertextuels) étudiés par Riffaterre sont toujours de l'ordre des microstructures sémantico-stylistiques à l'échelle de la phrase, du fragment...etc." ('82:9). Genette reçoit une telle impression apparemment à partir d'une lecture de l'article "La trace de l'intertexte" (Riffaterre 1980c). Il est vrai que la définition de l'intertexte -ou des rapports intertextuels- tend à varier selon l'article de Riffaterre consulté, et que dans 1979b ("Sémiotique intertextuelle: l'interprétant") Riffaterre utilise comme intertexte un deuxième texte dont la structure ne s'applique qu'à une partie du texte primaire (Maldoror II, 15, de Lautréamont). Il faut poursuivre la lecture suffisament loin dans Semiotics of Poetry pour trouver une définition de l'intertexte: "The other text enlightens the reader through comparison: a structural similarity is perceived between the poem and its textual referent despite their possible differences at the descriptive and narrative levels" (R. 1978:99-100; R.'83:130). Notons que la traduction française de 1983, à laquelle Genette se réfère, omet le terme essentiel de "structural". Car il ne s'agit jamais de citations ou d'autres ressemblances d'ordre lexical. Riffaterre souligne ce point dans sa définition en français cette fois- de 1979b: "tout rapprochement intertextuel sera réagi, imposé, non par des coïncidences lexicales, mais par une identité structurale, le texte et son intertexte étant des variantes de la même structure" (1979b: 132; c'est Riffaterre lui-même qui souligne). Il est dommage que Riffaterre s'égare quelquefois de cette définition, où il est clair qu'il s'agit de relations entre textes entiers.

18 En choisissant un modèle intertextuel pour corroborer notre hypothèse sur la structure matricielle du texte primaire, le plus facile sera de chercher parmi les textes appartenant à la même collection poétique, ou parmi d'autres ouvrages du même auteur. L'intertexte choisi par Riffaterre pour sa structure unimatricielle des Chats, par 
exemple, est justement Les Hiboux, qui suit notre sonnet dans Les Fleurs du Mal. Dans ce dernier poème l'immobilité 'contemplative' de ces oiseaux -que Riffaterre considère comme analogue aux caractéristiques des savants austères de notre texte- constitue le noyau conceptuel d'une matrice modèle de la forme "happiness is in sedentariness" (R. 1966:241).

La complexité que nos modifications ont apporté à l'intertexte de Riffaterre résultera en une réduction de la gamme de textes susceptibles de fonctionner comme modèle intertextuel pour le texte primaire.

20 Dans le présent cas, il faut que l'intertexte contienne l'idée - qui relie nos matricesde contraste entre deux mondes. Par exemple, il existe des serments bouddhistes de structure homologue, où c'est le rôle de la méditation de permettre à l'adepte de se libérer de l'existence corporelle pour atteindre un état équivalent au "rêve sans fin" de notre texte. Dans l'oeuvre de Baudelaire, l'on trouve assez souvent que l'un ou l'autre des deux mondes des Chats existe indépendamment; mais, dans au moins un autre poème, ils coexistent d'une façon analogue. Ce poème est Le Chat (Fleurs du Mal no.51), que Riffaterre lui-même cite, en notant que sa structure sémiotique est parallèle à celle des Chats -mais il n'en utilise que la partie qui convient à sa matrice 'contemplative'; il s'occupe plutôt de démontrer que la relation entre le chat et la femme que Jakobson impute aux Chats est également absente de ce poème (Riffaterre 1966:266). Le Chat aussi se divise - sur la plan graphique aussi bien que sémiotique- en une partie où il s'agit d'attributs physiques, et une autre où le chat devient une sorte d'être divin qui contemple son maître (voir les vers 21-24 et 35-40). Dans les deux cas, la contemplation serait le facteur permettant au chat de servir de médiateur entre le monde physique et le monde métaphysique avec ce résultat que le chat "se promène [...] dans ma cervelle[...] Ainsi qu'en son appartement" (vers 1-2).

21 Concernant la question du décalage chronologique qui peut exister entre texte et intertexte, à partir de Semiotics of Poetry (1978) il semble qu'en principe l'intertexte doit être antérieur au texte. Deux ans plus tard, Riffaterre nous dit simplement qu'il s'agit " de rapports entre une oeuvre et d'autres, qui l'ont précédée ou suivie " (Riffaterre1980c, p.4). En fin de compte, au delà de toute différence chronologique, la condition préalable de l'intertextualité est le fait que la structure sémiotique des deux textes doit être analogue.

22 Si l'on admet la possibilité d'un intertexte postérieur au texte, on ouvre la porte en principe à des interprétations de tendance plutôt 'post-moderne' -dans le sens de laisser plus de latitude au lecteur individuel. Il en serait besoin, peut-être, puisque peu de lecteurs ne possédant l'érudition d'un Riffaterre- il semblerait plus généreux de leur permettre de chercher des intertextes où ils voudraient. Toutefois, il importe de respecter la structure sémiotique du texte primaire et de ne pas se laisser tenter de lui imposer une structure intertextuelle non conforme.

Intertexte et récit

23 Considérons l'application de l'intertextualité chez Riffaterre au cas du texte narratif. Depuis un article de 1982 sur le romancier anglais Trollope, on trouve souvent la tendance à affirmer que sa définition de la littérarité -qui se base sur les caractéristiques de la poésie moderne- devrait s'appliquer à la littérature tout court. Dans un article plus récent (“Contraintes intertextuelles", 1997) Riffaterre parle dès le début de "définir la littérarité de la littérature", tout en utilisant une terminologie provenant de ses recherches antérieures sur la poésie. Par exemple, les types 
d'agrammaticalité paraissant dans cet article s'apparentent à ceux mentionnés dans Riffaterre 1978 à propos de la poésie -voire "les tropes, figures et symboles", auxquels il ajoute "les licences poétiques" (p.35). Les deux types d'intertexte mentionnés d'abord sont illustrés par application à des textes poétiques. Mais lorsque nous en venons au troisième -l'intertexte 'proleptique'-, nous avons l'admission de Riffaterre "qu'il s'agit d'une adaptation de l'intertextualité au narratif, en particulier dans le roman, dont la longueur exclut la possibilité d'un intertexte implicite qui serait coextensif au texte..." (p.49). Riffaterre semble donc se rendre compte de l'incompatibilité entre l'intertexte riffaterrien - par défintion coextensif au texte- et son application à la littérature narrative. La difficulté provient du fait que la structure matricielle (si elle existe) du texte narratif - qui serait à la base de toute intertextualité- fonctionnera sur le niveau intra-textuel. Cette 'matrice narrative' prend le plus souvent chez Riffaterre la forme d'un 'subtexte'.

24 L'on se rappellera que dans son article de 1990, "On Narrative Subtexts" ( R.1990g), Riffaterre a démontré l'existence d'un subtexte-voire d'une structure matricielledans la Recherche du temps perdu, de Proust, archétype du récit long. Il s'agit de la structure symbolique de la lanterne magique, qui se reproduit en une série de variantes tout au long du récit. Ce subtexte n'étant pas le seul dans la Recherche, l'on hésiterait de revendiquer, comme Riffaterre, que celui de la lanterne reflète, tout seul, la structure du récit dans son ensemble.

25 Dans son livre de la même année 1990 (Fictional Truth), la position de Riffaterre semble moins extrême. Là-dedans, il offre la définition suivante: "subtexts [are] texts within the text that are neither subplots nor themes but diegetic pieces whose sole function is to be vehicles of symbolism. They offer a rereading of the plot that points to its significance in a discourse closer to poetry than to narrative" (R.1990: xvii). Riffaterre semble donc envisager des mécanismes dont la fonction est d'engendrer des minipoèmes au niveau intra-textuel d'un récit narratif.

26 L'existence de plusieurs subtextes dans un texte narratif impliquerait la présence de la multi-matricialité dans un tel texte. Répétons, pourtant, que même dans le cas de multiples subtextes il semblerait hasardeux de proposer que ces derniers puissent correspondre exactement, dans l'ensemble, à la structure sémiotique globale d'un texte narratif.

L'interprétant

27 J'ai déjà remarqué que Riffaterre considère que la structure matricielle du texte constitue un signe textuel. Selon la version riffaterrienne de la triade de la signification (celle formulée par le philosophe américain Charles Peirce et désormais devenue classique), l'interprétant du texte (en tant que signe-sujet) et de l'intertexte (signeobjet) serait une deuxième structure quasi-intertextuelle capable de "médiatiser la référence du texte à son intertexte" (Riffaterre1980c, p.9). Ajoutons qu'il ne s'agit pas nécessairement d'une structure intertextuelle préexistante, comme le suggère Riffaterre dans 1986a (un article entitulé “Textuality"). L'interprétant consiste plutôt en une idée servant de signe médiateur, qui surgit chez le lecteur, l'aidant au processus de choisir un intertexte. Riffaterre décrit ce troisième signe médiateur comme "un présupposé qui empêche le texte de n'être que la répétition indifférenciée de son intertexte" (1980c, p.10).

28 Puisque l'interprétant comprend ces éléments de la structure sous-jacente du texte qui déterminent le choix d'un intertexte, son vocabulaire sera plus généralisé que celui de 
la structure matricielle du texte. L'on songe à l'exemple riffaterrien du "chameau qui porte son fardeau à travers la vaste étendue du désert", dont l'analogue intertextuel serait "le navire qui transporte son chargement d'un bout de l'océan à l'autre"propositions qui se basent sur la notion médiatrice généralisée d'une "véhicule robuste qui transporte sûrement son chargement / ses passagers à travers une étendue vaste et dangereuse". Cette dernière structure est quasi-intertextuelle en ce qu'elle pourrait s'appliquer à plus d'un autre texte -mais elle demeure "un présupposé...qui surgit chez le lecteur" durant sa recherche d'un intertexte.

L'interprétant doit être "formulé par le lecteur" pour une deuxième raison, que je n'esquisserai que brièvement. Lorsqu'il en vient à décrire le mécanisme selon lequel le texte moderne effectue son message novateur, j'ai proposé (dans mon livre de 1994) qu'il existe un "contexte sociolectique" dont le vocabulaire ressemble à celui de l'interprétant. Il s'agit d'un quasi-hypogramme déjà existant dans l'esprit du lecteur. Cependant, la relation entre ses deux concepts matriciels n'est pas le même que dans l'interprétant -ce qui produit un contraste sur le plan sémantique. L'interprétant est donc une paire de matrices reliées dont la relation interne contredit celle du contexte, produisant un choc aux préconceptions du lecteur. Il s'agit du fameux "choc du moderne", dont le résultat est de bousculer ses préconceptions, le conduisant à une nouvelle conception de ces aspects du monde dont le texte s'occupe.

\section{BIBLIOGRAPHIE}

Culler, J. 1981 The Pursuit of Signs. London, Routledge.

Genette, G. 1982 Palimpsestes. Paris, Seuil.

Hendricks, W.O. 1969 “Three models for the description of poetry”. Journal of Linguistics 5.

Hopkins, J.A.F. 1994/5, Présentation et critique de la théorie sémiotique de Michael Riffaterre. Tokyo, Sophia University Press.

Jakobson, R. 1981 Selected Writings III. The Hague, Mouton.

Riffaterre, M.1966 "Describing Poetic Strucures", Yale French Studies 36-37; traduction française in Essais de stylistique structurale. Paris, Seuil, 1971.

-1978 Semiotics of Poetry. Bloomington, Indiana Univ. Press (trad. française J.-J. Thomas, 1983.

Paris, Seuil).

-1979b “Sémiotique intertextuelle: l'interprétant". Revue d'esthétique 5. Paris, Collection 10/18.

-1980c, "La trace de l'intertexte", La Pensée 215

-1982 “Trollope's Metonymies”, Nineteenth Century Fiction. Univ. of California Press.

-1990 Fictional Truth. Baltimore \& London, Johns Hopkins U.P.

-R.1990g “On Narrative Subtexts”, in éds Caws \& Nicole, Reading Proust Now. New York, Peter Lang. 
-1997 “Contraintes intertextuelles”, in éds Le Calvez \& Canova-Green, Texte(s) et intertexte(s). Amsterdam, Atlanta GA, Rodopi.

\section{AUTEUR}

\section{JOHN HOPKINS}

Université de Tamagawa (Japon)

Ouvrage publié : Présentation et critique de la théorie sémiotique littéraire de Michael Riffaterre, Tokyo, Sophia University, 1994, 393 p., ISSN 0287-5357 